\title{
Improve Effective Capacity and Lifetime of Solid State Drives
}

\author{
Ping Huang, GuangPing Wan, Ke Zhou ${ }^{凶}$, Miaoqing Huang ${ }^{\S}$, Chunhua Li, and Hua Wang \\ Wuhan National Lab for Optoelectronics, Huazhong University of Science \& Technology (HUST), Wuhan,China \\ ${ }^{\S}$ Department of Computer Science and Computer Engineering,University of Arkansas, USA \\ Email: pinghp.hust@gmail.com,wan_guangping@126.com, \{k.zhou,hwang,li.chunhua $\} @$ hust.edu.cn,mqhuang@uark.edu
}

\begin{abstract}
Flash-based SSDs are becoming increasingly popular in modern storage systems, especially in high-performance computing infrastructures. However, several inherent technical limitations still remain to prevent their widespread deployment. One of the critical concerns is their limited lifetime, which is directly relevant to the total writes experienced by SSDs. In this paper, we present a Content and Semantics Aware File System (CSA-FS) which is able to reduce write traffic to SSDs. It employs deduplication and delta-encoding techniques to file system data blocks and semantic blocks, respectively. It is motivated by two important observations: (1) there exists a huge amount of content redundancy within primary storage systems, and (2) semantic blocks are visited much more frequently than data blocks, with each update bringing very minimal changes. By separately deduplicating redundant data blocks and delta-encoding similar semantic blocks, CSA-FS can significantly reduce the total write traffic to SSDs and greatly improve their lifetime correspondingly, at an acceptable cost of at most $7 \%$ performance degradation across a variety of workloads.
\end{abstract}

Keywords-File system; Solid State Drives; Deduplication; Delta-encoding

\section{INTRODUCTION}

Flash memory, which has traditionally been used in limited situations, has now become prevalent in modern storage systems in the form of flash-based SSDs thanks to their ever-expanding capacity and continuously decreasing cost. Because of their performance advantages, especially high random read performance, SSDs have been shown to improve the overall system performance greatly [1-3], especially in high-performance data intensive infrastructures [4] and transaction processing environments [5]. However, SSDs suffer from several inherent technical limitations. One of the most critical limitations is the their limited lifespan $[6,7]$, i.e., SSDs can only sustain a limited number of erase cycles $[8,9]$. Their storage would wear out and become unreliable once the threshold is passed [10]. The wear-out of SSDs is mainly caused by both external write traffic and internal write amplification [11]. While the total amount of external write traffic is determined by workloads, the write amplification is caused by page migration associated with internal Garbage Collection (GC) process [8].

Researchers have suggested a variety of effective techniques aiming to make the best of SSDs while avoiding their inherent limitations. Effective on-flash cache replacement algorithms [12] have been shown to improve small random write request performance and consequently reduce the amount of write amplification. Hybrid architectures composed of HDDs and SSDs [2,13] are also effective in extending the lifespan of SSD by directing most of the write operations to HDDs. More recently, deduplication technique has been integrated into the SSD Flash Translation Layer (FTL) $[14,15]$ to eliminate duplicate block writes. This integration has resulted in enlarged effective capacity and extended lifespan of SSDs.

In this paper, we propose a new scheme called Content and Semantics Aware File System (CSA-FS) to improve SSD's effective capacity and lifetime from system design perspective, which is very different from those existing studies. CSA-FS is a modified version of the Linux ext3 file system. Based on two important observations exhibited by realistic workloads (detailed in Section II), we propose to exploit workloads content commonality and similarity to reduce the write traffic. First, there widely exist duplicate contents in storage systems, both in backup/archival scenarios [16] and in primary storage systems [17-20]. Second, the block access patterns exhibit significant skewness due to the temporal and spatial locality of workloads [21]. Given the observed fact that semantic block operations account for a significant percentage of the total operations of workloads $[22,23]$, it is reasonable to assume that semantic blocks are hot blocks. Therefore, it is tempting to treat semantic and data blocks with different techniques.

CSA-FS applies deduplication to data blocks and deltaencoding to semantic blocks, respectively. Semantic blocks are extracted from the file system and exported for lookups. Semantic blocks mainly include super-blocks, group descriptors, data block bitmap, inode bitmap and inode tables. For every block write request, CSA-FS checks whether it accesses semantic block or data block by consulting the exported semantic blocks. For data block write, it computes its MD5 digest and looks up the hash value in a hash table to determine whether it is a duplicate block write. If it is a duplicate write, CSA-FS simply returns the block number in the found hash entry, and then uses that block number to update the block pointer table of the file's inode (with Linux ext3 file system, data blocks are normally accessed via their parent inodes). If it is a new write request, it first goes through the normal procedure, i.e., allocating a free block, updating the corresponding bitmap block and performing 
necessary accounting statistics, and finally inserts a new entry containing the block number, its MD5 value and some housekeeping information to the hash table. For metadata block write, CSA-FS calculates the content delta relative to its original content, and then appends the delta to a delta-logging region. By dealing with the semantic and data blocks differently, CSA-FS is able to eliminate duplicate data block writes and save a large amount of hash table memory, which would otherwise be used to host hash table entries corresponding to repeatedly changed metadata blocks if only deduplication technique had been deployed. CSAFS has been experimentally shown to have less than $7 \%$ performance overhead at worst and save up to $77 \%$ SSD capacity for our examined workloads, which has important implication for SSD capacity and lifespan.

It should be noted that thanks to SSD's high random read performance and being free from rotational latency, implementation of CSA-FS on SSDs would not cause fragmentation problems, which would otherwise cause severe problems on HDDs, especially in contexts of sequential access patterns. That is because file block deduplication would by nature cause indirected accesses (i.e., deduplicated blocks are very likely to be shared by multiple parent inodes) and introduce additional seek latency in the case of HDDs [19].

The main research contributions of this paper are twofold: (1) By analyzing real world workload traces, we make interesting observations that duplicate blocks often come from data blocks and semantic blocks are constantly changing. (2) Based on that observations, we propose an SSDoriented file system CSA-FS, which applies deduplication and delta-encoding to data blocks and semantic blocks, respectively, greatly suppressing SSD write traffic and correspondingly enhancing its reliability without incurring severe performance loss. To the best of our knowledge, this is the first work to reduce write traffic to SSDs at system level.

The rest of this paper is organized as follows. In Section II, we present the background and motivation. In Section III, we discuss related work. The design and implementation of CSA-FS is discussed in great detail in Section IV. In Section $\mathrm{V}$, we evaluate CSA-FS in respects of performance and its effectiveness in reducing the write traffic and improving lifetime by both benchmarking and simulation. Then we make a brief discussion about CSA-FS in Section VI and finally conclude the paper in Section VII.

\section{BACKGROUND AND MOTIVATION}

As the flash memory technology has been continuously advancing for the recent past years, flash-based SSD's capacity has greatly enlarged and its price has dropped dramatically, making it comparable to or even better than traditional HDD in terms of performance/cost. However, their inherent limitations have not been well resolved by manufacturers. On the contrary, the lifetime and reliability issues have become even worse as the number of bits stored in each storage cell increases and gradually approaches the limits of semiconductor physics. The common way to improve the lifetime and reliability of SSD is to reduce the write traffic arriving at SSDs. Currently, this is primarily achieved by suppressing writes $[14,15]$ or optimizing the access patterns arriving at SSD [2, 13].

The temporal and spatial locality of workloads have long been identified and deployed to optimize underlying storage systems. Recently, another locality called value locality has also been observed and utilized to improve I/O efficiency $[15,17]$. Value Locality implies that there exists a large amount of same block content in the requests, i.e., Value Popularity, and if a certain value is accessed, then it will be referenced again in the near future with a high probability, i.e., Temporal Value Locality. In addition, there are usually very few bit differences (i.e., content delta) between consecutive accesses to the same block, which has been successfully deployed to reduce storage requirements [24] or to optimize SSD access patterns [2]. It has been reported that semantic block operations represent a large portion of the total operations in both modern file systems and largescale storage systems [22, 23]. For example, in two enterprise deployed network file systems, it has been reported that about $21 \%$ of requests are file $\mathrm{I} / \mathrm{O}$ and about $50 \%$ are semantic block operations [23]. Surely, semantic block operations would cause their responsible semantic blocks to be accessed much more frequently than data blocks. Thus, the question to be asked is that how the temporal, spatial and value locality would be reflected in the access patterns of semantic blocks and data blocks? Is it possible to collectively deploy all of the three features to better reduce write traffic to SSDs?

To obtain an understanding of how block contents are accessed and distributed, we carried out a multi-dimensional analysis of the real world traces collected from production systems which were actively used at the FIU Computer Science department [17]. Those trace files record the disk I/O activities underlying ext3 file systems on top of which different workloads were running. They are representatives of daily primary workloads. Each of the contained record entry represents an individual $\mathrm{I} / \mathrm{O}$ and contains several pieces of information, including disk location, access time, responsible process id, MD5 digest, etc. For write reduction analysis, we separated out the writes by removing all read requests in the traces and only examined write requests. We present the webmail workload as our study example. Other workloads exhibit similar behaviors. More details about those traces can be found in [17]. The examined trace file contains a record number of about $450 \mathrm{~K}$ requests, which accessed 82,384 unique locations in total. Of the $450 \mathrm{~K}$ block writes, only $200 \mathrm{~K}$ different blocks of data were written, which implies that ideally we can achieve an impressive $55 \%$ block deduplication efficiency. 


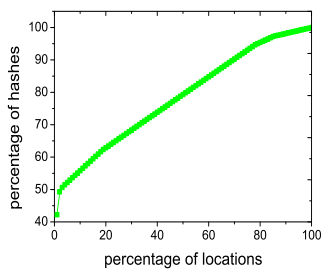

(a) The accessed percentage of locations VS the percentage of their responsible block writes relative to the total block writes.

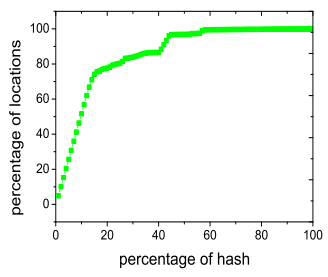

(b) The percentage of block writes VS the percentage of their accessed locations relative to the total locations.

Figure 1. In Figure 1(a), the most accessed locations are accounted in first, and then the less accessed ones, and so on. In Figure 1(b), the least popular block writes are accounted in first, then the more popular ones, and so on.

The analysis is intended to answer the following two questions: (1) How do the different locations contribute to the block values of the trace file (spatial locality)? and (2) What is the unique hash values' distribution pattern (value locality) among the locations? For that purpose, we sorted the write requests twice in terms of locations and hash values (i.e., block content), respectively. For sorting by location, we arranged them in descending order of the corresponding number of hash values, i.e., the number of requests that have ever been mapped to the same locations. For hash value sorting, we arranged them by the corresponding frequencies, i.e., how many times the individual hash values have ever appeared in the trace file, in ascending order. By analysis, we have made the following observations: (1) A small portion of the location space is intensively written, while the other portion is rarely written, This observation is also in line with the access skewness reported in previous study [21]. Figure1(a) and Figure1(b) show how locations absorb writes and how writes are distributed among the entire address space, respectively. Figure1(a) clearly demonstrates that a very small part of the locations are intensively overwritten again and again, causing them to account for a huge percentage of the hash values (i.e., distinct block content that needs storing). For example, more than half of the distinct block values, over $100 \mathrm{~K}$, were written to $3 \%$ of the locations, and the rest of locations have absorbed the remaining hash values equally, which is reflected by the smoother part of the curve. Figure1(b) shows the view from a different angle. As it is shown, the $65 \%$ most frequent hash values from the bottom of the sorted list (hash value sorting) only enlarges the accessed space by $10 \%$ new locations, which implies that locations were overwritten many times. Interestingly, the hash values experienced by the same location are different from each other most of the time. For instance, in our analysis, we found that the hottest location has experienced 2,050 hash values (block writes), of which 2,018 unique hash values have been identified, which means the same locations were most of the time overwritten with different contents.

(2) Hash values themselves exhibit significant skewness, i.e., one-shot hash values (singly occurred) account for a large percentage of the total hash values and exhibit distribution regularity, while a portion of the hash values were extremely popular. Of the $200 \mathrm{k}$ unique hash values, we have identified up to $140 \mathrm{~K}$ one-shot hash values, accounting for a percentage of $70.2 \%$. Furthermore, Figure 2 graphically shows the accessed locations of those one-shot hashes. From the figure, we know that one-shot hash values also tend to be concentrated on several hot regions. Table I lists the 10 most frequent hash values (i.e, most popular block content). It also clearly shows that the popularity of hash values are highly skewed, i.e., duplicate block writes exhibit quite different popularities. This phenomenon bears optimization opportunity, e.g., we can use less memory for hash table to host the most frequent hash values without losing much deduplication efficiency.

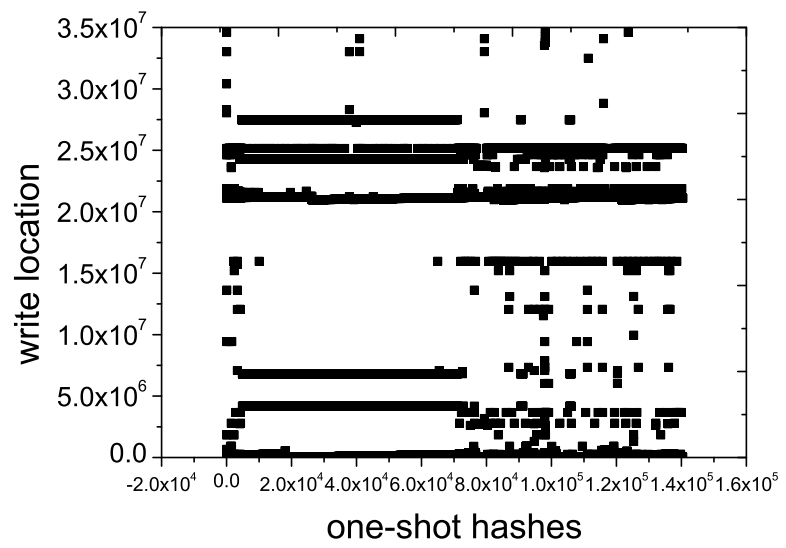

Figure 2. The distribution of the location set accessed by all of the one-shot hash values.

Summarizing the above analysis, we are able to reach the following three insights: (1) There exists a huge amount of content redundancy even in local storage systems; (2) A small part of the accessed space is intensively overwritten and consequently accounts for a significant percentage of all the hash values; (3) One-shot hash values represent an extraordinarily large portion of the block writes and most of them result from several hot regions being overwritten with different content. To leverage these observations, first of all, we can eliminate those write redundancy using deduplication technique. Second of all, previous researches have revealed that the overwriting content to the same location is minimally different from its original content [2, 24], which in this context means that the majority part of the unique hash values are very likely caused by repeatedly overwritten locations, because block updating would normally result in different block content. If we are going to single out those locations and separately apply delta-encoding [2, 24, 25] 
technique to them, then we would be able to trade deltaconsumed space for the space consumed by most of the oneshot hash values, which is surely much larger than the former (because in deduplication systems each distinct hash value represents a new block content and requires storage space) and thus it will be especially beneficial to SSD capacity and lifetime. Another advantage is that the memory requirement for deduplication hash tables would be significantly reduced due to the removed need to manage those separated oneshot hash values, which helps mitigate the main bottleneck of deduplication systems $[18,26]$ or equivalently improve the scalability of deduplication systems.

Table I

THE TOP-TEN MOST POPULAR HASH VALUES

\begin{tabular}{|c|c|c|c|}
\hline Hash Value & Frequency & Hash Value & Frequency \\
\hline Top1 & 6384 & Top6 & 3307 \\
Top2 & 6174 & Top7 & 3281 \\
Top3 & 5696 & Top8 & 2339 \\
Top4 & 5686 & Top9 & 801 \\
Top5 & 5668 & Top10 & 248 \\
\hline
\end{tabular}

\section{RELATED WORK}

CSA-FS is mainly based on deduplication and deltaencoding techniques. We discuss the related work of deduplication and delta-encoding.

Deduplication: Deduplication is a lossless storage compression that is able to reduce the storage space or the amount of network transmission. The basic idea behind deduplication is to store only one copy of identical blocks or to eliminate the need to transfer duplicate blocks. In deduplication systems, blocks are identified by its contenthashed signatures, for instance, MD5 or SHA-1 hash values. LBFS [27] breaks the contents into variable-length blocks in a content-defined manner and computes their hash values as the identifers to eliminate the requirement of transferring redundant contents over network, greatly saving the network bandwidth. In CSA-FS, duplicate blocks are identified by their MD5 digest over fix-sized file system blocks. Many efforts have been devoted to address the diskbased fingerprint-lookup problem to fasten the deduplication process and improve its throughput. DDFS [26] is a production deduplication file system implemented by Data Domain. Sparse indexing [16] exploits the inherent locality within backup streams to avoid storing the full index. Instead, it only keeps sampled fingerprints. Guo et al. [28] deployed three techniques named progressive sampling index, grouped mark-and-sweep and client pipelining to improve the singlenode scalability for deduplication. Meyer et al. [29] studied the feasibility and efficacy of data deduplication by analyzing hundreds of realistic file systems content. However, deduplication has previously often been conducted in the contexts of backups or archival systems, which exhibit good locality and similarity. Recently, researchers have studied deduplication in primary workloads. Koller [17] found the existence of block content similarity and utilized that to improve I/O performance and reduce the mechanical delays. CA-SSD [14, 15] augments the SSD Flash Translation Layer (FTL) [30] with deduplication functionality to reduce the actual write traffic to flash medium, which greatly enlarges their effective capacity and prolongs their effective lifetime. Although bearing similar philosophy as CA-SSD, CSA-FS works at the file system layer sitting atop of SSDs instead of being implemented at block level within SSD.

Delta-encoding: Researchers have observed that many data intensive applications exhibit strong content locality, which means that block write operations typically bear only $5 \%$ to $20 \%$ bit changes relative to the old content [24]. Those small changes are called deltas relative to their old content and can be delta-encoded to save storage space. TRAP [24] is a CDP (Continuous Data Protection) scheme that deploys delta-encoding to only store the deltas of changing blocks. It has been shown to significantly reduce the required storage requirement. Specifically, for each incoming write request, it performs XOR operation between the old content and the new one, and then compresses the zero-dominated XORed results as deltas. I-CASH [2] intelligently couples HDD and SSD to form a hybrid I/O architecture, with SSD storing the seldom-changed reference blocks and HDD logging the deltas between currently accessed blocks and the corresponding reference blocks. By doing this way, I-CASH is enabled to take advantage of the complementary merits of HDDs and SSDs. Difference Engine [31] employs deltaencoding to improve the memory page-sharing efficiency among different virtual machines and provides up to a factor of 1.5-2.5 memory-savings for various workloads at a cost of less than $7 \%$ performance overhead. In the same manner, CSA-FS also uses delta-encoding specifically to metadata blocks to suppress write traffic to SSD.

\section{System DESIGN AND IMPLEMENTATION}

Considering the semantic blocks-intensive nature of workloads [22, 23], the first reasonable approach to identifying those possible hot regions that are mainly responsible for one-shot block content (see the discussion in Section II) is to assume semantic blocks as hot regions. Based on this assumption, we designed a proof-of-concept prototype of CSA-FS. We chose ext3 as our base file system. Figure 3 shows the architectural overview of CSA-FS. As it is shown in the figure, CSA-FS has added four functionality modules into its base ext3 file system counterpart. It partitions the underlying SSD into Data Region and Shaded Region. Data Region is the region that is exported to CSA-FS. Shaded Region is used as delta logging region and persistent storage space for hash tables. Semantics Parser is a module that is loaded into the kernel to parse the on-disk file system layout. Its purpose is to export a look-up table recording all the 
semantic block address information for future consultation to determine whether the incoming write request is headed to semantic blocks or data blocks. DedupEngine is responsible for duplicate data blocks detection and suppression. DeltaEngine computes the content deltas of semantic block writes and hands them to Delta Logger. Delta Logger caches the small deltas generated by DeltaEngine, groups together the deltas associated with those semantic blocks in the same block group and periodically flushes them to the delta logging region. In the following subsections, we discuss each of the components in more detail.

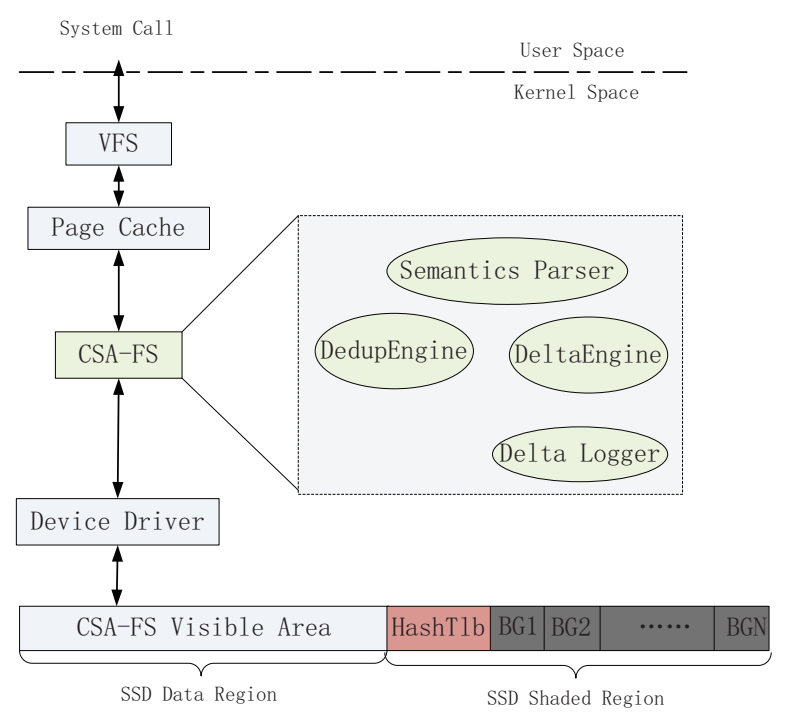

Figure 3. CSA-FS Architectural Overview.

\section{A. Semantics Parser}

Disk blocks have different importance imposed by the represented file system, and usually exhibit different access behaviors. File system semantic blocks are those blocks describing the file system structure and organization. Prior researches have already demonstrated that they can be deployed to realize special purposes $[32,33]$. The functionality of Semantics Parser is to identify those semantic blocks and exports them for future reference when deciding whether a write is to semantic block or data block. Thanks to ext3's fixed structure, it is easy to identify its semantic blocks once ext3 has been created on a disk. Though there are other types of semantic blocks, Semantics Parser only exports those static semantic blocks, including superblock, group descriptors, data block bitmap, inode bitmap and inode table blocks. Starting from the superblock, all those semantic blocks can be easily identified using linear calculations.

\section{B. DeltaEngine}

DeltaEngine is specifically designed to handle the writes to semantic blocks. For each arriving write, it performs an exclusive-ored (XOR) operation between the new content and its original content so that the resultant block content would be zero-dominating due to the minor bit differences $[2,24]$. Then it uses zlib to compress the resultant block to a delta which is much smaller than a block. After that, it hands the delta and the reference block number to Delta Logger. To make the delta computing process fast, DeltaEngine caches a number of reference metadata blocks from the same block group using LRU algorithm. The heuristic is that semantic blocks in the same block group are expected to be accessed together. For instance, ext3 tends to allocate the subdirectories inodes in the same block group as their parent. There is additional treatment for data block bitmap. For data block bitmap, DeltaEngine tries to determine whether there are data blocks that the write is going to release (change the corresponding bits from 1 to 0 ) and add those to-be-released blocks number to a release-list, which is scanned by a space recycling thread. The block number identification is a three-step process. First, the new content is XORed with the old content. Second, the resultant block is ANDed with the old content. In this step, the $1 \mathrm{~s}$ in the final result exactly represent the original $1 \mathrm{~s}$ that have changed to $0 \mathrm{~s}$. For example, suppose the old content is $1010 \mathrm{~b}$ and the new content is $1001 \mathrm{~b}$, implying the first and second bits have been flipped over. Then the intermediate result is $1010 \mathrm{~b} \oplus 1001 \mathrm{~b}=0011 \mathrm{~b}$, and the final result is $0011 \mathrm{~b} \& 1010 \mathrm{~b}=0010 \mathrm{~b}$, indicating the second bit has been flipped over from 1 to 0 . Lastly, translate the bit positions within the final resultant block to global block numbers.

\section{Delta Logger}

Deltas generated by DeltaEngine actually represent small changes to semantic blocks and are typically very small in size. Writing those small-sized deltas directly to SSD is especially harmful [12]. Rather than writing deltas immediately to underlying SSDs, Delta Logger buffers the deltas first, then periodically flushes them in batches using append mode to the shaded logging region later on when the buffered deltas accumulate to be of multiple pages in size, which is friendly to SSD. Delta Logger segregates the buffer memory into different small buckets, each of which is responsible for hosting deltas associated with semantic blocks within the same block group. The buckets are periodically flushed to the delta logging regions. As illustrated in Figure 3, the SSD delta logging region is correspondingly divided into different small regions. When the available space of the small region falls below a preset threshold (e.g., 20\%), all of the deltas in that region are scanned to be coalesced with their respective reference blocks to generate newest semantic blocks, and the delta logging region is reclaimed for future reuse. At the same time, if the newest semantic blocks are cached in DeltaEngine for delta computing reference, DeltaLogger signals DeltaEngine 
to invalidate them. The reason why we divide the logging region by block groups is that block groups usually exhibit access locality as mentioned in the above subsection and as a consequence they would cause their corresponding SSD logging memory to be consumed quickly and thus would be flushed onto SSD logging region timely. Upon reads to a semantic block, all the buffered deltas belonging to the semantic block are flushed and the corresponding SSD logging region is coalesced with the reference block to generate the latest content.

\section{DedupEngine}

DedupEngine intercepts the data block write and uses the writing content's MD5 value to guarantee its uniqueness. In ext3, for every file data block write, it scans the appropriate data block bitmaps to find a free block. Next, it writes the content to the chosen block, sets the corresponding bit to indicate the block has been used and finally updates some statistics-related fields. In CSA-FS, this procedure is trapped and modified to achieve deduplication purpose as follows. First, the MD5 hash value of the writing block content is calculated. Then, the MD5 value is attempted to be searched in a hash table which maps MD5 values to block addresses where the actual content is stored. Third, if this MD5 is not found in the hash table, meaning that this is a new block content, it defaults to the normal block write procedure, after which it inserts a new hash entry containing the corresponding information including MD5 value, block number, reference counter and other auxiliary information into the hash table with the reference counter initially being set to 1. Finally it inserts a pair of 〈number, hashindex $\rangle$ into a second hash table which maps block numbers to indexes to facilitate the space reclaim thread as discussed next. On the other hand, if a hash entry with the same MD5 value already exists, it simply increments the entry's reference counter, signals write completion to the writer and returns the block number associated with the hash entry.

In conventional backup/archival deduplication systems, reclaiming storage space was rarely considered as a primary concern [26]. However, with CSA-FS, data blocks that have been released by the upper layer must be freed to the system. To accomplish this, a reclaiming thread is scheduled to be initiated periodically to perform the reclaiming operation. Data blocks releasing is inferred by DeltaEngine through inspecting the data block bitmap content modification and related information is passed on to the reclaiming thread through release-list as discussed previously. The reclaiming thread starts to work by examining the release-list, and for each of the block addresses on the list, it looks it up in the second hash table to obtain their indexes into the first hash table. After that, it uses those indexes to access the corresponding hash entries and decrement the reference counter. If the reference counter drops to zero, it resets the block's indication bit in the block bitmap to indicate that the block is freed and can be reused. The whole deduplication process is totally oblivious to data block read, because the block address table in inodes has always been storing the block number where the actual content are stored.

\section{E. Putting it All Together}

Putting it all together, Figure 4 shows the CSA-FS workflow handling block requests. For every incoming request, it first decides whether the request is a semantic block request or a data block request by searching the visiting address in the semantic block table exported by Semantics Parser. Then it identifies whether it is a read or write request. For data block read, it defaults to the original data read path; for data block write, it goes to DedupEngine which computes the MD5 of the writing content and uses the MD5 to determine whether this is a new block content or a duplicate one. If it is a new block, it writes the content to a newly allocated block and updates the hash tables. Otherwise it just updates the reference counter of the corresponding hash entry and returns. For semantic block write, it goes to DeltaEngine which calculates the changed content between the new content and the old content, appends the resultant delta to the corresponding logging bucket and flushes the bucket to SSD delta logging region if the triggering threshold is satisfied. For semantic block read, it first flushes the deltas residing in the corresponding bucket and then coalesces the delta logging region with its corresponding semantic block, then returns the updated block content and invalidates its version in DeltaEngine if it was cached as reference block.

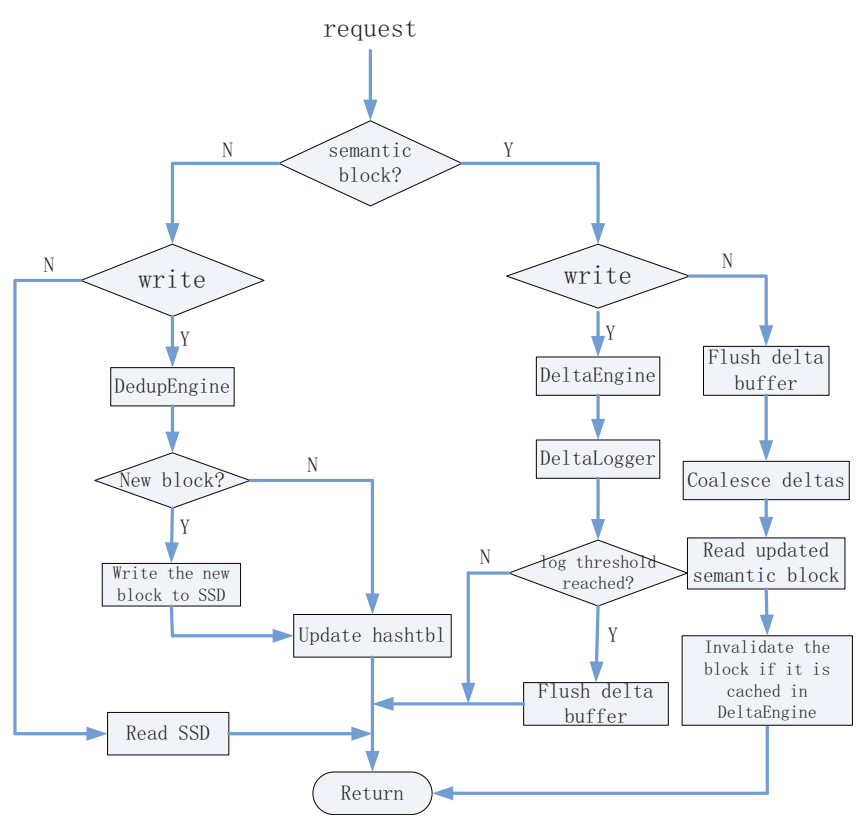

Figure 4. CSA-FS Workflow Overview 


\section{Evaluation}

In this section we present the extensive experiment to evaluate CSA-FS. The experiments mainly follow two steps. First, we ran the chosen benchmarks and tests on both CSAFS and the unmodified ext3 file system counterpart (denoted as vanilla thereafter). We reported the results in terms of performance, CPU utilization and specifically the effects of delta-coding technique. Second, we used the collected blocklevel traces of the experiments to feed an SSD simulator [8] to specifically study the efficacy of CSA-FS in improving capacity and lifetime of the underlying SSDs.

\section{A. Experimental Setup and Workloads}

We implemented a proof-of-concept prototype of CSAFS. The test hardware platform features two dual-core Intel Xeon $(\mathrm{R})$ processors and $8 \mathrm{~GB}$ memory. The operating system is Centos6.5 with Linux kernel 2.6.32. We used a Kingston MLC 60GB SSD on which we created ext3 and CSA-FS respectively to run the tests. The SSD was connected to the server through a RAID card interface. We adopted TPC$\mathrm{C}$, TPC-W and kernel building as target workloads. TPC-C and TPC-W are representative online transaction processing workloads, which require high-performance. TPC-C is a commonly used benchmark that models the operational end of businesses where real-time transactions are processed. It simulates the execution of a set of distributed and online transactions (OLTP) against data warehouses. We use one of the TPC-C implementations written by Hammerora Project [34] and build data tables for 5 warehouses with 25 users issuing transactional workloads following the TPC$\mathrm{C}$ specification. TPC-W, is a transactional web benchmark developed by Transaction Processing Performance Council that models an on-line bookstore. It typically simulates the on-line consumer behaviors, including web-browsing, shopping, order-processing, etc. We use the Java TPCW implementation from University of Wisconsin-Madison [35] to build an experimental environment. The underlying database for both TPC-C and TPC-W is MySQL 5.0 and the reported performance is transactions per second. Kernel building is the building operation of Linux kernel 2.6.24 and its performance metric is reported by the time elapsed during the compiling process. To avoid cache pollution, we unmounted and remounted the file systems each time before the experiment start. The file systems were mounted using default format and mount options.

\section{B. Performance Comparison}

In this section, we give a performance comparison of the three workloads on ext3 and CSA-FS. To ensure the figures to be put together in one picture, we report the CSA-FS performance normalized to the performance of ext3. Figure 5 shows the performance comparison. As it is shown, for TPC-C and TPC-W, CSA-FS performs better than vanilla, with $12 \%$ and $14 \%$ performance improvement, respectively.
But for Kernel Building, CSA-FS slightly underperforms vanilla, with a minimal degree of $7 \%$ performance degradation. The reason why TPC-like workloads get performance improvement is because TPC-like workloads are dominated by random patterns and database table operations would typically cause partial block overwrites/updates, which were well captured by DeltaEngine in CSA-FS. DeltaEngine translates them to sequential write and saves a lot of I/Os because of the adopted logging mechanism. But for kernel building, since the intermediately generated files are almost new and different from each other, the computing overheads associated with the MD5 calculation, delta-computing and delta-coalescing on reads have offset the benefits of saved I/Os, causing the overall performance slightly degraded.

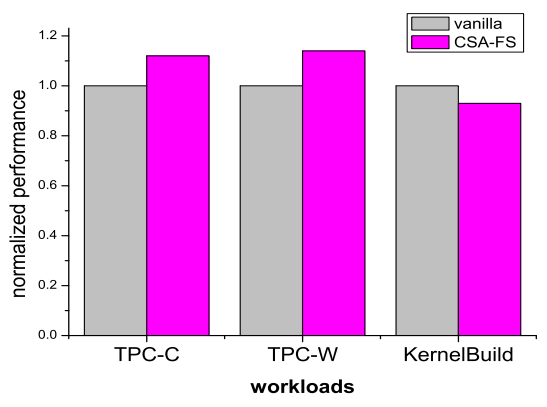

Figure 5. Normalized workloads performance comparison on vanilla ext3 and CSA-FS file systems.

\section{CPU Utilization}

Due to the introduction of MD5 digest computation and delta-encoding operation in CSA-FS, it is obvious that CSAFS would be more CPU-intensive than vanilla ext3. To verify this, we present the average CPU utilization during the respective running phases in Figure 6. As demonstrated, compared with ext3, CSA-FS has consistently increased CPU utilization by a degree of $17 \%, 22 \%$ and $28 \%$, for TPC-C, TPC-W and KernelBuild, respectively, implying that CSA-FS has traded CPU cycles for improved capacity usage efficiency and prolonged lifetime as discussed in the following subsections. Another important observation is that the overall CPU utilization is very low in ext3 for all of the three workloads, only even at most $30 \%$ for KernelBuild which is characterized as computation-intensive workloads. In other words, CPU is largely under-utilized and I/O should be the bottleneck requiring improvement. Thus, trading CPU cycles for I/O performance is realistically viable, especially when the CPU computing power has been growing significantly with the emergence of multicore processors. Actually, one of the enabling reasons that make primary storage inline deduplication [19] practical and viable is the big performance gap between CPU and the I/O subsystems. 


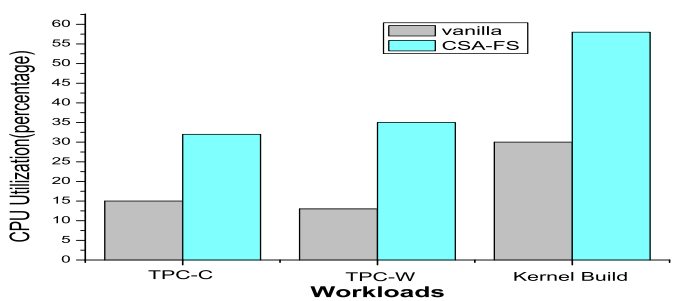

Figure 6. The average CPU utilization of the running experiments on ext3 and CSA-FS file systems.

Table II

THE NUMBER OF HASH ENTRIES

\begin{tabular}{|c|c|c|}
\hline Workloads & dedupfs & CSA-FS \\
\hline TPC-C & $65 \mathrm{~K}$ & $12 \mathrm{~K}$ \\
\hline TPC-W & $48 \mathrm{~K}$ & $17 \mathrm{~K}$ \\
\hline KernelBuild & $111 \mathrm{~K}$ & $59 \mathrm{~K}$ \\
\hline
\end{tabular}

\section{Delta-encoding Contribution}

In this section, we discuss the effects of delta-encoding semantic blocks in CSA-FS. We compare CSA-FS with its variance called dedupfs, in which all data blocks and metadata blocks are dealt with indistinguishably using deduplication. Then we compare the number of hash entries (i.e., distinct block content) associated with them. Table II shows their respective number of hash entries. As clearly shown, CSA-FS has significantly less hash entries for all of the three workloads than dedupfs, e.g., for TPC-C workloads, the number of hash entries in CSA-FS is only one-fifth that of dedupfs. The significant reduction in the amount of hash entries has three important implications. (1) It successfully demonstrates the effectiveness of delta-encoding semantic blocks and validates our initial assumption that semantic blocks are repeatedly updated, causing a lot of new blocks. (2) Each hash entry represents a distinct block content that needs to be stored in the SSD. Therefore, the greatly reduced hash entries would translate to significantly saved storage requirement, as we see in Section V-E. (3) Hash table memory is correspondingly reduced. Hash index lookup is critical to the performance and scalability of deduplication systems and has long been criticized as their bottleneck. In CSA-FS, the significantly reduced memory for hash entries is realized only at a cost of a configurable amount of deltas-logging memory, which can be flexibly configured depending on the available memory in the system.

\section{E. Space Saving}

In this section and the following section we report the simulation experimental results to demonstrate how CSA-FS has successfully fullfilled our goal. We used the blktrace [36] tool to record the block $\mathrm{I} / \mathrm{O}$ activities occurred during the experimenting processes. Then we fed the traces from both ext3 and CSA-FS to an SSD simulator from Microsoft [8] to study the SSD internal behaviors. We simulated a 60GB MLC SSD and used the default SSD simulator parameters as in [8]. This subsection presents how much data were written to the used SSD during their respective running experiments and the next subsection discusses how the amount of reduced write traffic affects SSD's lifetime.

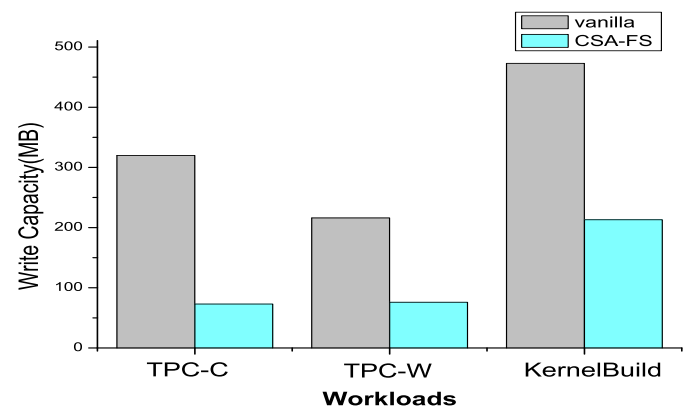

Figure 7. The consumed SSD capacity by the workloads.

One useful indicator that can be utilized to characterize the lifetime of a given SSD is the total amount of external write traffic the SSD has experienced. Because from a long term perspective, unlike conventional HDDs, an SSD can only sustain a fixed amount of write traffic during it entire life. Thus, the less write traffic it experiences, the less wear-out the corresponding workload has imposed on it. To calculate the total consumed space, we sum up the request size of all of the write trace requests. Figure 7 shows the workloads' consumed SSD capacity. As depicted, CSA-FS is able to save a great deal of space. Specifically, CSAFS saves up to $77 \%, 65 \%$ and $55 \%$ write traffic for TPC$\mathrm{C}$, TPC-W and KernelBuild, respectively. The saved space comes from the elimination of identical block writes and small delta writes as replacement of whole semantic block writes. In summary, thanks to the employed deduplication and delta-encoding techniques, CSA-FS is able to enlarge the SSD's effective usage capacity for a variety of workloads by a very impressive extent.

\section{F. Improved Lifetime}

Finally, to quantify to what extent the SSD is actually worn out, which is related to both external write traffic and internal write amplification, we report the number of erase operations occurred during the workloads running phases. Table III shows the results. It clearly demonstrates that compared with the original ext3 file system, CSA-FS performs much less erase operations, due to the reduced write traffic as discussed in the preceding section. In addition, as SSDs wear out, the reliability degrades. As a result, CSA-FS has 
Table III

THE NUMBER OF ERASE OPERATIONS

\begin{tabular}{|c|c|c|c|}
\hline & ext3 & CSA-FS & Saved \\
\hline TPC-C & 2347 & 636 & $73 \%$ \\
\hline TPC-W & 1896 & 589 & $69 \%$ \\
\hline KernelBuild & 3672 & 1324 & $64 \%$ \\
\hline
\end{tabular}

also improved SSD reliability. Overall, CSA-FS can improve the SSD lifetime and reliability over ext3 file system.

\section{DISCUSSION}

As observed in prior researche, workloads are often sort of semantic blocks-intensive, which would translate to widely different access behaviors between semantic blocks and data blocks. In general, semantic blocks are accessed much more aggressively, especially when the file system is used in synchronous mode which is considered good to system reliability. Apparently, any updates to a block would cause its content and correspondingly its MD5 to be different. So we know for sure that the MD5 of the changing semantic blocks would also be changing. Furthermore, as demonstrated by the trace analysis, the semantic blocks contribute the most part of the one-shot hash values, which means the changing of semantic blocks would enlarge the total number of hash values significantly. In other words, if we do not consider those semantic blocks to be hashed, then the number of hash values can be significantly reduced and memory requirement for hash table could be correspondingly reduced, mitigating the RAM bottleneck problem especially associated with primary data deduplication systems [18]. The more important heuristic is that calculating MD5 digests of metadata blocks for the purpose of deduplication does not make any sense but only waste CPU power, because they would surely generate distinct MD5 digests due to its being changed, which has been verified by the reduced hash entries in our experiments.

\section{CONClusion And Future Work}

We have presented an SSD-oriented extension to the Linux ext3 file system, which is called CSA-FS. CSA-FS is intended to greatly reduce the write traffic to SSD by applying deduplication to regular data blocks and deltaencoding to semantic blocks. It was based on the observations that data blocks exhibit reasonable redundancy and semantic blocks are modified much more frequently with minimal differences each time but contribute a very large portion of the total amount of hash values. Our experimental results with a variety of workloads have demonstrated that CSA-FS is capable of reducing up to $77 \%$ write traffic and correspondingly improving reliability, at a cost of higher CPU utilization and marginal performance impacts, $7 \%$ degradation at worst.
Further directions for continuing research include the following. First, Semantics Parser can only export the information about static metadata blocks. There are other kinds of dynamic meta blocks, for example, indirect and direct entry blocks. We plan to develop more sophisticated mechanisms that can determine those dynamic metadata blocks on-thefly. For example, it would be helpful to pack detailed request information from upper layer into the block request for the decision process [33]. Second, while semantic blocks are kind of hot regions that bear the characteristics observed in Section II, we want to explore other types of hot data regions [37] to investigate whether there exists similar opportunity for optimization. Third, currently, CSA-FS does not take the journal block layer into account, we plan to extend it to handle journal block layer as well.

\section{ACKNOWLEDGMENT}

The authors would like to thank the anonymous reviewers for their constructive comments which greatly improve the correctness and readability of this paper. The work is supported in part by the National Natural Science Foundation of China under Grant No.61232004, the National Basic Research Program (973 Program) of China under Grant No.2011CB302305, the National Science and Technology Support Program under Grant No.2012BAH35F03-03, and the US NSF under grant CNS-1219062. Any opinions, findings, and conclusions expressed in this paper are those of the author(s) and do not necessarily reflect the views of the supporting agencies.

\section{REFERENCES}

[1] F. Chen, D. Koufaty, and X. Zhang, "Making the best use of solid state drives in high performance storage systems," in Proceedings of the 25th International Conference on Supercomputing(ICS'2011), 2011.

[2] J. Ren and Q. Yang, "I-cash: Intelligently coupled array of ssds and hdds," in Proceedings of the 17th IEEE International Symposium on High Performance Computer Architecture (HPCA'2011), 2011.

[3] G. Wu, X. He, and B. Eckart, "An adaptive write buffer management scheme for flash-based ssds," ACM Transactions on Storage(TOS), vol. 8, no. 1, 2012.

[4] A. M. Caulfield, L. M. Grupp, and S. Swanson, "Gordon: using flash memory to build fast, power-efficient clusters for data-intensive applications," in Proceedings of 14th International Conference on Architectural Support for Programming Languages and Operating Systems(ASPLOS'09), 2009.

[5] S.-W. Lee and B. Moon, "Design of flash-based dbms: an in-page logging approach," in Proceedings of the 2007 ACM SIGMOD international conference on Management of data(SIGMOD’2007), 2007.

[6] V. Prabhakaran, M. Balakrishnan, J. D. Davis, and T. Wobber, "Depletable storage systems," in Proceedings of the HotStorage'2010, 2010.

[7] G. Wu and X. He, "Reducing ssd read latency via nand flash program and erase suspensions," in Proceedings of the 10th USENIX Conference on File and Storage Technologies(FAST'2012), 2012. 
[8] N. Agrawal, V. Prabhakaran, T. Wobber, J. D. Davis, M. Manasse, and R. Panigrahy, "Design tradeoffs for ssd performance," in Proceedings of USENIX Annual Technical Conference, 2008.

[9] Y. Hu, H. Jiang, L. Tian, H. Luo, and D. Feng, "Performance impact and interplay of ssd parallelism through advanced commands, allocation strategy and data granularity," in Proceedings of the 25th International Conference on Supercomputing(ICS'2011), 2011.

[10] L. M. Grupp, A. M. Caulfield, J. Coburn, and S. Swanson, "Characterizing flash memory: Anomalies, observations and applications," in Proceedings of the 42nd International Symposium on Microarchitecture(MICRO'2009), 2009.

[11] X.-Y. Hu, E. Eleftheriou, R. Haas, I. Iliadis, and R. Pletka, "Write amplification analysis in flash-based solid state drives," in Proc. of the Israeli Experimental Systems Conference(SYSTOR'2009), 2009.

[12] H. Kim and S. Ahn, "Bplru: A buffer management scheme for improving random writes in flash storage," in Proceedings of the 6th USENIX Conference on File and Storage Technologies(FAST'2008), 2008.

[13] G. Soundararajan, V. Prabhakaran, M. Balakrishnan, and T. Wobber, "Extending ssd lifetimes with disk based write caches," in Proceedings of the 8th USENIX Conference on File and Storage Technologies (FAST'2010), 2010.

[14] F. Chen, T. Luo, and X. Zhang, "Caftl: A content-aware flash translation layer enhancing the lifespan of flash memory based solid state drives," in Proceedings of 9th USENIX Conference on File and Storage Technologies (FAST'2011), 2011.

[15] A. Gupta, R. Pisolkar, B. Urgaonkar, and A. Sivasubramaniam, "Leveraging value locality in optimizing nand flashbased ssds," in Proceedings of 9th USENIX Conference on File and Storage Technologies (FAST'2011), 2011.

[16] M. Lillibridge, K. Eshghi, D. Bhagwat, V. Deolalikar, G. Trezise, and P. Camble, "Sparse indexing: large scale, inline deduplication using sampling and locality," in Proccedings of the 7th USENIX conference on File and storage technologies(FAST'2009), 2009, pp. 111-123.

[17] R. Koller and R. Rangaswami, "I/o deduplication: Utilizing content similarity to improve i/o performance," in Proceedings of the 8th USENIX Conference on File and Storage Technologies (FAST'2010), 2010.

[18] A. El-Shimi, R. Kalach, A. Kumar, A. Oltean, J. Li, and S. Sengupta, "Primary data deduplication - large scale study and system design," in Proceedings of the 2012 USENIX Annual Technical Conference(USENIX ATC'2012), 2012.

[19] K. Srinivasan, T. Bisson, G. Goodson, and K. Voruganti, "idedup: Latency-aware, inline data deduplication for primary storage," in Proceedings of the 10th USENIX Conference on File and Storage Technologies (FAST'12), 2012.

[20] D. Meister, J. Kaiser, A. Brinkmann, T. Cortes, M. Kuhn, and J. Kunkel, "A study on data deduplication in hpc storage systems," in Proceedings of the International Conference for High Performance Computing, Networking, Storage and Analysis(SC'2012), 2012.

[21] M. Bhadkamkar, J. Guerra, L. Useche, S. Burnett, J. Liptak, R. Rangaswami, and V. Hristidis, "Borg: Block-reorganization for self-optimizing storage systems," in In Proceedings of the 7th File and Storage Technologies Conference(FAST'2009), 2009.

[22] A. W. Leung, M. Shao, T. Bisson, S. Pasupathy, and E. L. Miller, "Spyglass: Fast,scalable metadata search for largescale storage systems," in Proceedings of the 7th USENIX Conference on File and Storage Technologies(FAST'2009), 2009.
[23] A. W. Leung, S. Pasupathy, G. Goodson, and E. L. Miller, "Measurement and analysis of large-scale network file system workloads," in Proceedings of the 2008 USENIX Annual Technical Conference, 2008.

[24] Q. Yang, W. Xiao, and J. Ren, "Trap-array: A disk array architecture providing timely recovery to any point-in-time,' in Proc. of the 33rd Annual International Symposium on Computer Architecture(ISCA'06), 2006.

[25] C. B. M. III and D. Grunwald, "Peabody: The time travelling disk," in Proc. of IEEE Mass Storage Conference, 2003.

[26] B. Zhu, K. Li, and H. Patterson, "Avoiding the disk bottleneck in the data domain deduplication file system," in Proceedings of the 6th USENIX Conference on File and Storage Technologies (FAST'08), 2008.

[27] A. Muthitacharoen, B. Chen, and D. Mazières, "A lowbandwidth network file system," in Proceedings of the 18th ACM Symposium on Operating Systems Principles (SOSP'01), 2001

[28] F. Guo and P. Efstathopoulos, "Building a high-performance deduplication system," in Proceedings of the USENIX ATC'2011, 2011.

[29] D. T. Meyer and W. J. Bolosky, "A study of practical deduplication," in Proceedigns of the 9th USENIX conference on File and storage technologies(FAST'2011), 2011.

[30] J.-Y. Shin, Z.-L. Xia, N.-Y. Xu, R. Gao, X.-F. Cai, S. Maeng, and F.-H. Hsu, "Ftl design exploration in reconfigurable high-performance ssd for server applications," in Proceedings of the 23rd International Conference on Supercomputing(ICS'2009), 2009.

[31] D. Gupta, S. Lee, M. Vrable, S. Savage, A. C. Snoeren, G. Varghese, G. M. Voelker, and A. Vahdat, "Difference engine: Harnessing memory redundancy in virtual machines," in Proceedings of the 8th USENIX Symposium on Operating System Design and Implementation(OSDI'2008), 2008.

[32] H. S. Gunawi, V. Prabhakaran, S. Krishnan, A. C. ArpaciDusseau, and R. H. Arpaci-Dusseau, "Improving file system reliability with i/o shepherding," in Proceedings of the 21 st ACM SIGOPS Symposium on Operating Systems Principles(SOSP'07), 2007.

[33] G. Sivathanu, S. Sundararaman, and E. Zadok, "Type-safe disks," in Proceedings of the 7th Symposium on Operating Systems Design and Implementation(OSDI'2006), 2006.

[34] “http://hammerora.sourceforge.net/."

[35] M. H. Lipasti, "Java tpc-w implementation distribution," http://pharm.ece.wisc.edu/tpcw.shtml.

[36] http://linux.die.net/man/8/blktrace.

[37] A. Wildani, E. L. Miller, and L. Ward, "Efficiently identifying working sets in block i/o streams," in Proceedings of the 4th Annual International Systems and Storage Conference(SYSTOR'2011), 2011. 